\title{
Research on the Problem of Seamless Integration of Virtual Reality in Augmented Reality System
}

\author{
Feng Guo ${ }^{1, a}$, Fangwei $\mathrm{Mo}^{2, \mathrm{~b}}$ and Gang $\mathrm{Wu}^{3, \mathrm{c}}$ \\ 1, 2, 3 Wuhan Mechanical Institute, Wuhan, China \\ a294244476@qq.com, b165007605@qq.com, ‘9429208@qq.com
}

\section{Keywords: Augmented reality; Seamless fusion; Virtual reality; Alpha channel technology}

\begin{abstract}
Augmented reality technology is a new technology developed on the basis of virtual reality technology, which is a new development of virtual reality technology. The key of augmented reality technology is to integrate the $3 \mathrm{D}$ virtual objects generated by computer with the objects in the real scene, so as to supplement and enhance the real world information. Augmented reality technology is involved in many fields of computer science. Most of the early researches focus on the problem of $3 \mathrm{D}$ registration. In recent years, with the deepening of the research, how to improve the visual consistency of the augmented reality system, and provide a more realistic and soft interface of virtual reality, has become a new research hotspot. In order to improve the sense of reality in augmented reality system for the purpose of synthetic images, proposes a new occlusion algorithm, this algorithm avoids the usual way in the past, but the operation mechanism of Open based on Inventor, the design of dynamic EMO node, and the virtual scene is divided into hierarchical modeling, while the use of moving target under complex background the extraction technology and Alpha channel technology, to achieve multi-level virtual real scene mutual occlusion. Since the algorithm does not need to model the real environment in advance, so a lot of preliminary work is reduced, and the versatility and portability of the system are improved. More importantly, the algorithm for non rigid objects, can also be a good completion of the virtual and real mutual occlusion.
\end{abstract}

\section{Introduction}

With the continuous development of AR technology, it has been widely used in engineering, education, medical, entertainment and other fields. At present, more and more, in the 3D registration AR system discussed domestic however, achieve seamless integration of the actual situation of the scene, so that the system has good visual consistency, is an important factor to promote the popularization and application of the AR technology as soon as possible to. Therefore, seamless integration, visual consistency is an urgent need for in-depth study. AR is related to many fields of computer science, including multimedia, three-dimensional modeling, real-time video display and control, sensor fusion, real-time tracking and 3D scene registration, fusion and many other new technologies and new; it is a kind of real world and virtual world information technology information seamless integration. In order to realize the seamless integration of virtual reality scene in AR system, this paper presents an algorithm to realize the multi layer occlusion relationship between virtual and real objects. This algorithm avoids both at home and abroad used prior to the real scene modeling method, using the method of moving object extraction and background of Alpha channel based on the combination, allows the system to automatically generate the occlusion mask features using the Open Inventor node tree, node dynamic mask design, finally realizes the mutual occlusion of the actual situation.

\section{The Realization of the Most Basic Fusion Effect}

The most simple, the most basic fusion effect, is a simple superposition of both before and after: the real scene of the video image as the background, three-dimensional virtual objects stacked before. The problem of mutual occlusion is not considered in the fusion process. 
Acquisition of Virtual Reality Scene. There are two kinds of data which are used for scene fusion in AR system: one is the real scene information (stereo video signal) and the other is virtual scene information (3D model). Video capture is a part of the whole scene acquisition system. We install the helmet on the left and right two cameras, as well as the two were installed in the two PC video capture card, to achieve this part of the signal acquisition. Taking into account the development of the platform is built on the basis of the Windows platform, and in the Windows platform DireetX DircctShow is a Windows video technology generic interface. It uses a framework to filter (filter) as the basic unit, through the video stream capture, compression / decompression, transmission, playback and a series of filters in series to achieve video applications [L03][L04]. The technology has good scalability, and the video capture card has good support. Therefore, we use the Directshow software technology for video capture and processing. Video capture OireetShow filter framework as shown in Fig. 1.

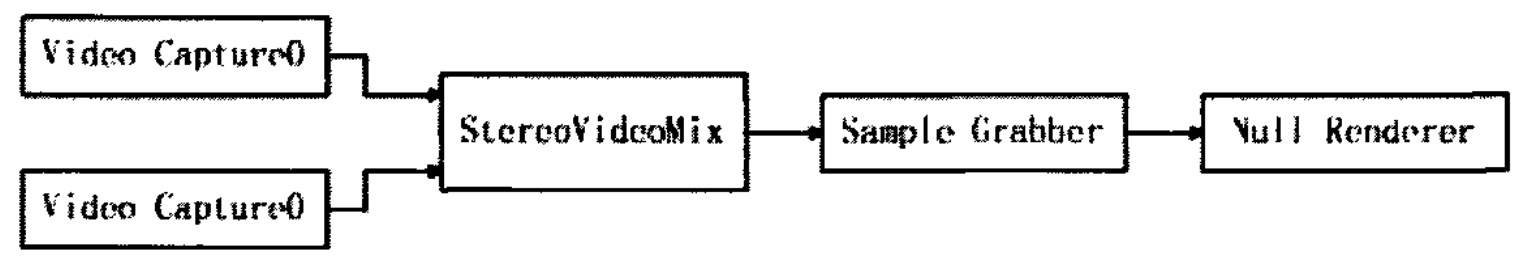

Figure 1. Video capture filter

A large part of the augmented reality system is aimed at the processing of virtual models. AR system requires real-time, dynamic and realistic simulation environment. Considering the limitation of hardware and the real-time requirement of virtual reality system, the modeling of AR system is different from that of the modeling method based on modeling. The modeling of AR mostly adopts the technology of model segmentation, texture mapping and so on. At present, there are two main ways to construct the virtual scene in AR: model based method and image based rendering (IBR) method. These two methods can be implemented and verified in 3D Max. In order to enable the AR system to produce a strong sense of reality in the vision, so as to realize seamless integration of virtual scene, we creatively interactive real-time 3D graphics modeling function and Open Inventor 3D Max drawing function combination and virtual environment has a strong realistic impact effect.

Key Techniques of Scene Composition. We developed the I1lusion platform, the need to achieve three-dimensional graphics processing. We use the SGI company Open Inventor development kit [W94][H03] 3D, this is a building on the OpenGL 3D graphics interface general commercial 3D development kit, consisting of a large class of complete written by $\mathrm{C}++$. Open Inventor toolbox is the core of Open Inventor, it not only provides users with a powerful programming interface, but also manage the OpenInventor created each object. It consists of three parts: 3D scene database, interactive operation and object component library. The 3D database contains a series used to construct 3D scene graph nodes and the corresponding function object; node library provides many predefined Inventor Open node component and collocation mechanism, users can according to their own needs to join the object matching mechanism to expand the node library; mainly uses very intuitive and interactive operation you can edit the response to the user of the scene graph interactive operation event. The node is the most fundamental part of object 3D scene database, hierarchical structure consists of nodes called the scene graph, the scene graph is not a simple combination of nodes, which defines the order of nodes, nodes impact on the back of the node appears earlier in the scene, at the same time, it also provides a mechanism to limit this effect thus, a part of the scene, can open with other parts separated in function. Nodes can be divided into 3 categories: shape node, attribute node, packet node. The geometry shape of node objects in the scene, including two categories: basic geometric shapes, such as cone, cuboid, sphere and cylinder; complex geometric shapes, such as patch set, square grid, line set, Nurbs surface and so on. The attribute node records the attributes that are applied to the geometric objects, including the material, texture, lighting, viewpoint and so on. Grouping nodes combine more than one node so that they can be treated as a single object. Common packet nodes include Group nodes, Separator 
nodes and Switch nodes. After two nodes are derived from the Group node, in addition to containing the geometry, attribute and grouping nodes like Group as nodes, Separator nodes can take effect within the group node constraints inside group, while the Switch node can display a serial number according to the individual nodes within the group. Each node has a corresponding range and method, by calling the method, you can set or modify the corresponding range. For example, the material node, can set the color of the light reflected from the geometry of the transmitted light, color, transparency and so on: grouping nodes, child nodes can add, delete node, change the ordering of child nodes and so on.

Fusion of Virtual Reality Scene. The final synthesis of our program is shown by Open Inventor, but in this environment, there is no direct video fusion method, so we need to solve the problem by other means. Through the research, we found that all nodes are derived from SoNode, which provides a virtual function OLRender a private SoNode, and a deeper look, every time Open Inventor when drawing the scene, all is according to the level of scene graph traversal, GLRender function call for each node to draw. The 6LRender function of the various nodes are pre packaged by the Open Inventor library, and the function is a private function, we can not change the mapping of a built-in node. Therefore, by changing the way the model is drawn, we still can not achieve the background display. Open Inventor can be extended based on the characteristics, we consider that: as long as the design of a node, the node to OLRender function to draw the real scene video, and the nodes in the scene graph to the top left, you can ensure that all the virtual scene drawing before the object is drawn to complete the background video. Based on this principle, we realize the fusion of real scene video and virtual objects. Open Inventor scene graph drawing is a node of a node in accordance with the order of priority in depth in order to render each node. Therefore, as long as the video image real scene display node before the node of each model, you can before rendering a 3D model for rendering real video images, the virtual object model in part of the video, virtual image synthesis and the basic. We will draw the background video node design named BackgroundVideo, as shown in Fig 2, the whole scene graph at the root node, the node subtree from left to right with the camera, video background emission node node and one other object. Open Inventor core first get the parameters of the camera in the drawing, and then use the background node representation of the real scene, then a description of all objects in the scene, and finally through the projection to calculate changes in the location of the camera projection image and to portray it.

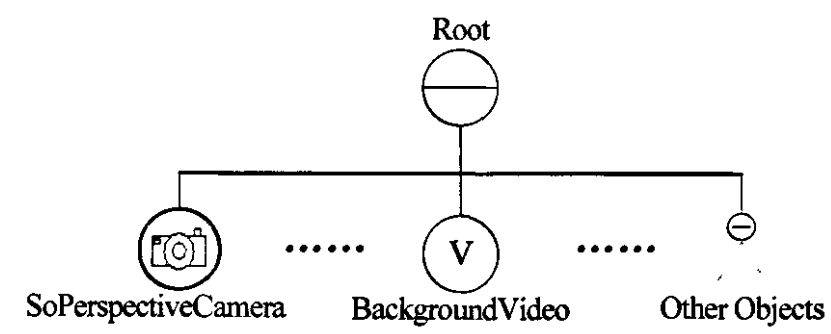

Figure 2. Virtual reality image synthesis module simple scene

\section{Virtual Reality Scene Image Decomposition}

Grouping Modeling of Virtual Scene. The virtual scene, before entering the AR environment, by the use of three-dimensional modeling software made in advance. In order to facilitate the control of occlusion in the fusion process, we consider the virtual objects in the environment according to a certain combination of decomposed into different units, and for each unit were established and were to be guided into the IV file. Of course, different AR systems have their own different application characteristics, we should give the unit partition scheme of virtual scene according to its characteristics. We carried out the AR system research, mainly for the indoor environment to expand the application of [FDG06][FCT06]. Taking into account the indoor environment within the scope of the occlusion is mainly caused by the user's point of view in the direction of the virtual reality of the spatial location of the staggered. Therefore, the object of the virtual scene is needed to 
be split into several units according to the depth of the user observation direction from the front to the back. For indoor decoration of our virtual system, the distance from the user view some furniture combination at the same level as a new unit, according to the rules, we will design the virtual furniture combined into 3 units, and the model, as shown in fig. 3. Here for the convenience of discussion, we will be three virtual units were recorded as VP1, VP2, VP3. This is ready for the following occlusion treatment.

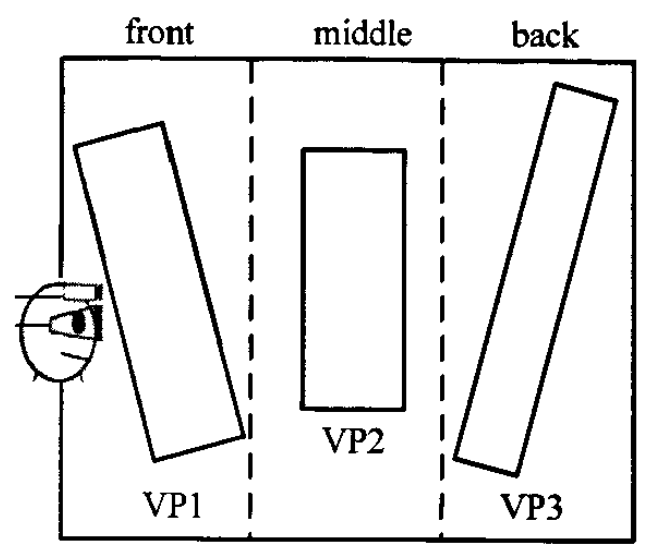

Figure 3. Split and reorganization of indoor virtual furniture

Division of Moving Objects in Real Scene Images. The virtual scene is completed before entering the AR environment. However, the real situation is different. The real scene image information is composed of video capture devices directly in the AR environment to obtain real-time, before the actual image fusion, real scene information is completely unknown, we do not have any prior knowledge of the. Therefore, the real scene can not be like a virtual scene, before entering the AR environment is decomposed into several units. Analysis of the indoor environment of the AR system and found that the real scene of the video image as the background and the virtual scene of the image fusion, usually can achieve better results. Only when some of the objects in the real scene move, so that their own spatial location changes, resulting in the space between the virtual and real objects to create a staggered, resulting in mutual occlusion relationship. Therefore, the moving objects in real scenes are key to creating a shielding phenomenon, we try to separate the moving objects from real video images, and as an independent unit of operation and control, virtual objects occlusion becomes possible. For the extraction of moving objects in real scene, we can use the technology of moving object extraction in complex background.

\section{Introduction of Alpha Channel}

In the simple fusion of virtual reality scene, the real scene is placed as a virtual object in the background. When an object in a real scene is moved to a part of the virtual object, the occlusion is produced. At this time the image of the moving object should be placed in front of the virtual object, thus showing the effect of occlusion between virtual and real objects. In order to make the OEI image can be effectively stacked with other images; we have processed the image to add the Alpha channel. The constituency is a representation of the Alpha channel, which can be used to create any shape of the Alpha channel. Since the establishment of the correct constituency is the first step in image processing, the processing of the constituency directly affects the final result. For the content discussed in this paper, the selection of the object is separated from the background image of the moving object area, we will be marked as MOA (Moving Object Areal). The contour of the moving object is extracted, and the whole OEI image is divided into two parts, one is the region in the contour, the MOA is selected, the outer area is composed of the second parts. 


\section{The Final Realization of Virtual Reality and Multi-layer Occlusion}

In order to realize the fusion of virtual and real images, the video images should be placed before the virtual 3D images. Since we decompose the virtual scene into a number of units (VP1, VP2, VP3), so we will render the composition of the composition of the scene design diagram shown in fig. 4.

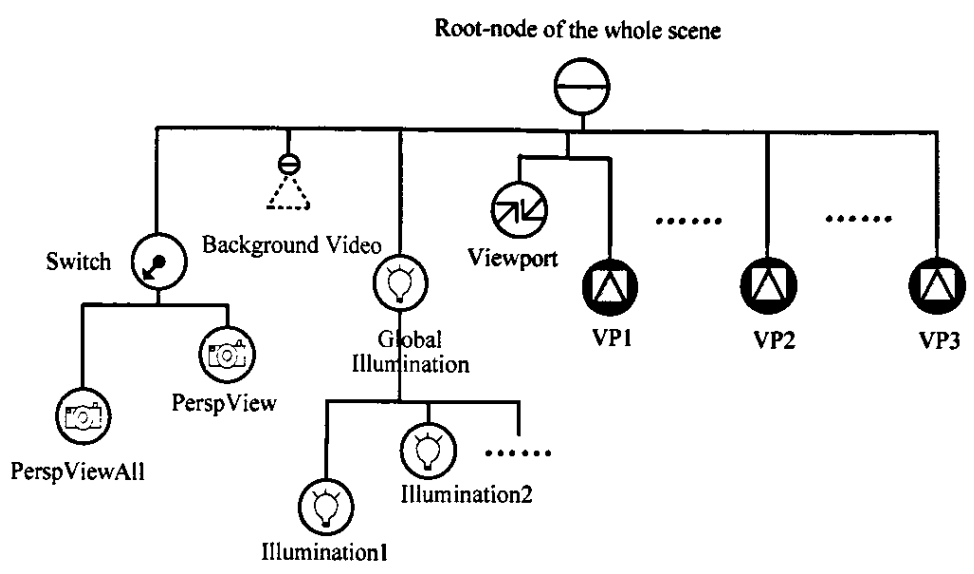

Figure 4. Scene tree structure after image decomposition

To set the OEI image of Alpha channel, in order to synthetic image fusion in real video and virtual objects, we use the 01 characteristics of ell Inventor scene tree, designed a mobile node effectively rendering objects, and named it MON (Moving Obstruct Node). The principle of the image of the node is similar to the above we designed the Background Video, through the GLRender function to complete the image display. The position of moving objects in real scene is random and unpredictable, so the occlusion relationship between the real and the real scene is also changing constantly. In order to synthesis image easily and accurately render the change of occlusion, we in the scene tree structure diagram of each virtual unit back, add a MON node, as shown in Fig. 5, respectively MON1, MON2, MON3. When the actual situation does not occur when the phenomenon of mutual occlusion, all MON nodes do not play a role, here we call it in a dormant state. When occlusion occurs, some MON nodes are activated according to the spatial location of the moving object".

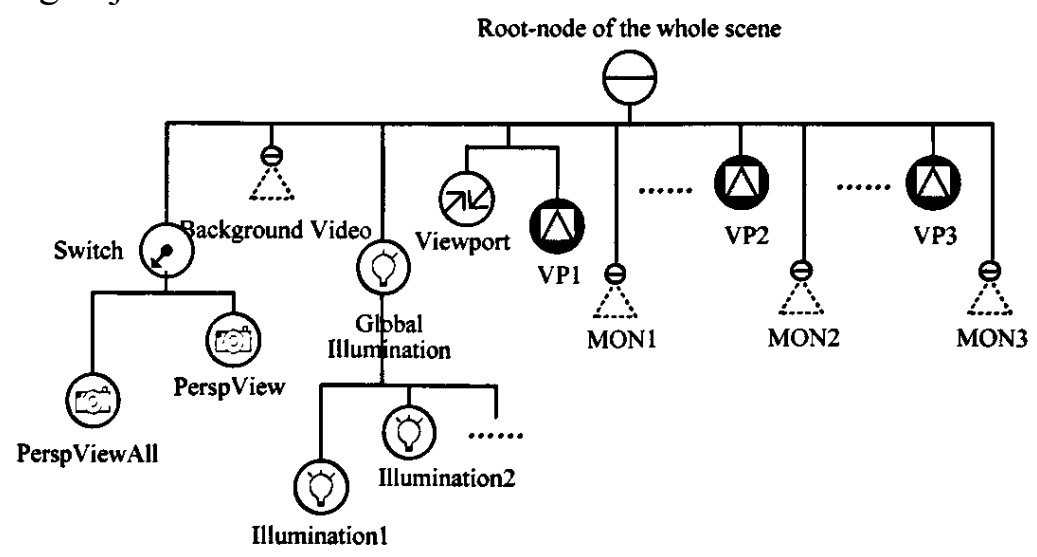

Figure 5. Scene tree structure after image decomposition

\section{Conclusion}

The correct representation of the mutual occlusion between virtual and real objects is an important means to realize the seamless integration of virtual reality scene and improve the visual consistency of AR system. In this paper, we discuss how to implement occlusion without any prior information of the real scene, and propose a new occlusion rendering algorithm. The Open method from the Inventor scalability of rendering node design of video image and ingenious arrangement of scene 
tree structure, and combined with the use of the moving target in complex background of the existing extraction technology, and make full use of the Alpha channel characteristics of the image, to achieve the multi virtual objects between occlusion. Since the algorithm does not need to know the real scene in advance, it is not necessary to model it, so it can greatly reduce the workload and can be used in any real environment. In addition, the algorithm uses the technology of moving object extraction, so it can be used to achieve the non rigid object occlusion.

\section{References}

[1] Aragon. Cecilia R. Usability evaluation of a flight-deck airflow hazard visualization system[C], Proceedings of A / AA / IEEE Digital Avionics Systems Conference, 2004:4. B. 2-1-4. B. 2-11.

[2] Altmann. J, Gubrud, M. Anticipating military nanotechnology[J].Technology and Society Magazine ,IEEE ,2004, 23(4); 33-40.

[3] Rosten, Edward, Reitmayr, Gerhard, Drummond, Tom. Real-time video annotations for augmented reality[C], Advances in Visual Computing-First International Symposium, ISVC2005, Proceedings, LNCS, 2005, 3804:294-302.

[4] Eric Foxlin, Extended draft version of Chapter of Handbook of Virtual Environment Technology [M],Lawrence Erlbaum Associates ,2002.

[5] F. Yan, Y.M. Chen. Rectification of magnetic force tracker using neural network in

[6] AR system[J] ,Journal of shanghai university ,2006, 10(5):431-435.

[7] F. Yan, Y.M. Chen and T. Wen. Augmented Reality with Multilayer Occlusion[C].

[8] Eurographics UK Chapter Proceedings of Theory and Practice of Computer Science

[9] 2006:99-104.

[10]F. Yan, Y.M. Chen and M.H. Wang, Multi-sensor Data Fusion Based on Fuzzy

[11] Integral in AR System[C] ,Proceedings of the 16th International Conference on artificial

[12] reality and telexistence ,2006:155-162. 